\title{
Aspirin inhibits the growth of Helicobacter pylori and enhances its susceptibility to antimicrobial agents
}

\author{
W H Wang, W M Wong, D Dailidiene, D E Berg, Q Gu, K C Lai, S K Lam, B C Y Wong
}

Gut 2003;52:490-495

\begin{abstract}
Background and aim: The role of Helicobacter pylori and aspirin in peptic ulcer formation and recurrence remains an important clinical topic. The interaction between aspirin and $H$ pylori in vitro is also not clear. We investigated the effect of aspirin on the growth of $H$ pylori and on the susceptibility of $H$ pylori to antimicrobials.

Methods: Time killing studies of $H$ pylori were performed with different concentrations of aspirin and salicylate. Growth of bacteria was assessed spectrophotometrically and by viable colony count. The effects of aspirin on the efficiency of colony formation and on metronidazole induced mutation to rifampicin resistance in $\mathrm{H}$ pylori were determined. Minimal inhibitory concentrations (MICs) of aspirin and metronidazole were tested by the standard agar dilution method. MICs of amoxycillin and clarithromycin were determined by the $E$ test method. Results: Aspirin and salicylate inhibited the growth of $\mathrm{H}$ pylori in a dose dependent manner and bactericidal activity was due to cell lysis. Aspirin $400 \mu \mathrm{g} / \mathrm{ml}$ caused a 2 logs decrease in colony forming units $/ \mathrm{ml}$ at 48 hours, and suppressed the normal ability of metronidazole to induce new mutations to rifampicin. The $\mathrm{IC}_{90}$ of aspirin was $512 \mu \mathrm{g} / \mathrm{ml}$. Increased susceptibility of amoxycillin, clarithromycin, and metronidazole to $\mathrm{H}$ pylori was observed at $1 \mathrm{mM}(180 \mathrm{\mu g} / \mathrm{ml})$ aspirin.

Conclusions: Aspirin inhibited the growth of $\mathrm{H}$ pylori, suppressed the mutagenic effect of metronidazole, and enhanced the susceptibility of $\mathrm{H}$ pylori to antimicrobial agents. This mechanism is important in future drug development for effective clearing and overcoming resistance.
\end{abstract}

See end of article for

Accepted for publication 4 November 2002

$\mathrm{H}$ elicobacter pylori and aspirin are the two most important causative factors in the pathogenesis of peptic ulcer However, the mechanism of interaction between $H$ pylori and aspirin has not been sufficiently documented to date, either in vitro or in vivo. In one study, the number of endoscopic mucosal lesions in patients taking low dose aspirin was higher in those with $H$ pylori infection than in those with no $H$ pylori infection. ${ }^{1}$ In another study, more antral ulcers occurred in $H$ pylori negative than in $H$ pylori positive subjects taking low dose aspirin. ${ }^{2}$ It is generally recognised that $H$ pylori infection does not worsen mucosal injury with aspirin or nonsteroidal anti-inflammatory drug (NSAID) usage because $H$ pylori infection is associated with increased mucosal levels of prostaglandins..$^{2-4}$ Although reports from clinical studies are controversial, $^{125}$ it seems that there is no meaningful difference in $H$ pylori prevalence between aspirin users and non-users. ${ }^{67}$ The background rate of infection in aspirin or NSAID users was high, ranging from $24 \%$ to $68 \%$, but therapy with aspirin or NSAIDs does not increase susceptibility to $H$ pylori infection. ${ }^{5-7}$

Previous studies have reported that aspirin and other NSAIDs interfere with growth of both Gram negative and Gram positive bacteria in vitro, such as Escherichia coli, ${ }^{8}$ Candida albicans, ${ }^{89}$ Staphylococcus saprophyticus, ${ }^{89}$ Pseudomonas cepacia, ${ }^{10}{ }^{11}$ and Staphylococcus aureus. ${ }^{12}$ In some organisms, increased resistance or susceptibility to several antibiotics can be induced by growing in a subinhibitory concentration of aspirin..$^{10-14}$ Graham and colleagues ${ }^{15}$ reported that $H$ pylori was not susceptible to aspirin and other NSAIDs, such as indomethacin, ibuprofen, naproxen, or tolmetin in vivo. However, Caselli and colleagues ${ }^{5}$ observed an anti- $H$ pylori effect of aspirin, diclofenac, and ketoprofen in vitro. Therefore, we undertook this study to evaluate the possible effect of aspirin on the growth of $H$ pylori and further determined the effect of this drug on susceptibility of $H$ pylori to antimicrobial agents.

\section{MATERIAL AND METHODS}

\section{Bacterial strains}

The experiments were conducted with 63 clinical isolates of $H$ pylori from ethnic Chinese in Hong Kong, reference strains NCTC 11637, NCTC 12908, and isogenic metronidazole susceptible (MtzS) and resistance (MtzR) derivatives of $H$ pylori 26695, containing rdxA::cam and frxA::kan single and double knockout mutations in the nitroreductase genes, and equivalent derivatives of SS1. The clinical isolates were obtained from patients undergoing upper endoscopy examination and were maintained frozen at $-80^{\circ} \mathrm{C}$ in brain heart infusion (BHI) broth (BHI; Oxoid, Basingstoke, UK) with $20 \%$ glycerol. Endoscopic diagnosis included normal appearing mucosa in 15 patients, duodenal ulcer in 30 patients, gastric ulcer in three patients, and gastric cancer in five patients. An additional eight $H$ pylori isolates from aspirin taking patients and two isolates from other NSAID taking patients were included in the study. Bacteria were re-plated on Columbia agar (Oxoid) supplemented with 7\% horse blood under microaerobic conditions produced by a gas generating system (CampyGen; Oxoid) for three days. A non-pathogenic $E$ coli strain (DH5a) was also included and cultured under similar conditions as described above in an aerobic ambient.

\section{Chemicals}

Aspirin (Sigma Chemical Co, St Louis, USA) was freshly dissolved in dimethylsulphoxide (DMSO; Sigma, USA) and

Abbreviations: MIC, minimal inhibitory concentration; NSAID, non-steroidal anti-inflammatory drug; MtzS, metronidazole susceptible; MtzR, metronidazole resistance; $\mathrm{BHI}$, brain heart infusion; FBS, fetal bovine serum; DMSO, dimethylsulphoxide; CFU, colony forming unit; RifR, rifampicin resistance. 
metronidazole (Fluka, Switzerland) was prepared in water. Sodium salicylate (Sigma Chemical Co) was dissolved in water.

\section{Establishment of growth in broth medium}

Time killing studies were performed using five different strains (NCTC 11637, NCTC 12908, 26695, one isolate from a duodenal ulcer patient, and one isolate from a gastric ulcer patient) in Brucella broth (Difco Laboratories, Detroit, Michigan, USA) supplemented with 10\% fetal bovine serum (FBS; Gibco-BRL, Grand Island, New York, USA). Culture media (20 $\mathrm{ml}$ ) in a set of $10 \mathrm{~cm}$ petri dishes with or without test drug were inoculated with $10^{8}$ colony forming units (CFU) $/ \mathrm{ml}$ of $H$ pylori. Dishes were placed in an anaerobic jar (Oxoid) and incubated at $37^{\circ} \mathrm{C}$ under microaerobic conditions, as described above, on a shaker at $90 \mathrm{rpm}$. A vehicle control of DMSO (less than $0.1 \%$ ) was included in the studies.

\section{Determination and adjustment of $\mathrm{pH}$}

The pH of Brucella broth supplemented with 10\% FBS before inoculation with or without aspirin was measured using a digital pH meter (HI 9024; Hanna Instrument, Portugal). Growth of H pylori NCTC 11637 in the presence of aspirin (400 $\mu \mathrm{g} / \mathrm{ml}$ ) was compared with that in the medium with the same $\mathrm{pH}$ adjusted by $1 \mathrm{~N} \mathrm{HCl}$ in the absence of the drug.

\section{Growth measurement}

Growth of bacteria in broth was assessed spectrophotometrically at $600 \mathrm{~nm}$ and by viable colony count. At 24 and 48 hours, samples were removed and serially diluted 10-fold, and colony counts were determined by plating $100 \mu \mathrm{l}$ of each dilution on duplicate agar plates that allow optimal growth of $H$ pylori. After 4-6 days of incubation under microaerobic conditions at $37^{\circ} \mathrm{C}$, the plates were read.

\section{Microscopy}

Undiluted samples from the above broth medium with either aspirin or vehicle, after incubation for 24 and 48 hours, were spread onto glass slides and examined under Gram stain by light microscopy.

\section{Determination of efficiency of plating}

Strains of $H$ pylori were inoculated on Columbia blood agar plates with various concentrations of aspirin $(0,100,200$ $\mu \mathrm{g} / \mathrm{ml})$ and metronidazole $(0,3,8,16,25,32 \mu \mathrm{g} / \mathrm{ml})$, and were incubated under microaerobic conditions at $37^{\circ} \mathrm{C}$ for three days. Wild-type and isogenic MtzS and MtzR derivatives of strain 26695, and rdxA::cam and frxA::kan single and double knockout mutant derivatives of strains 26695 and SS I were included in this study to test the possible effects on aspirin sensitivity of loss of nitroreductases encoded by rdxA and frxA, the activities responsible for susceptibility of $H$ pylori to metronidazole. ${ }^{16}$ The efficiency of plating was the titre of CFU obtained from the plates with the test drug divided by the titre obtained from the control plates without drug.

\section{Metronidazole induced mutation}

New mutations to rifampicin resistance (RifR), which result from changes in the rpoB gene, ${ }^{17}$ were quantified as a measure of metronidazole induced mutation in $\mathrm{H}$ pylori. Bacterial cells $\left(10^{7}-10^{8}\right)$ of isogenic MtzS and MtzR derivative of 26695 were spread onto Columbia agar plates containing various concentrations of metronidazole $(0,3,8,16,25,32 \mu \mathrm{g} / \mathrm{ml})$ and aspi$\operatorname{rin}(0,100,200 \mu \mathrm{g} / \mathrm{ml})$. Following three days of incubation, bacterial cells were suspended in phosphate buffered saline and spread on Columbia blood agar with $5 \mu \mathrm{g} / \mathrm{ml}$ rifampicin and titrated by spotting aliquots of serial 10 -fold dilutions on rifampicin free medium. Each test was repeated three times and average values were reported.

\section{MIC determination}

Bacteria were prepared in BHI to yield a viable count of $3 \times 10^{8}$ $\mathrm{CFU} / \mathrm{ml}$ (equivalent to $1 \mathrm{McF}$ arland turbidity standard unit), and used as the inoculum for susceptibility testing. The minimal inhibitory concentrations (MICs) of aspirin were determined by the agar dilution method according to NCCLS document M1 l-A2 on 66 strains of $H$ pylori, ${ }^{18}$ and the MICs of other antimicrobials (clarithromycin and amoxycillin) were determined by the E test method, according to the manufacturer's guidelines, on 24 strains (including three reference strains). MIC was recorded as the lowest concentration that inhibited visible growth of organisms and the results were determined after 72 hours of incubation at $37^{\circ} \mathrm{C}$ under microaerobic conditions.

For the agar dilution method, aspirin or metronidazole was diluted in distilled water and incorporated separately into Columbia blood agar. Plates contained twofold serial dilutions of drugs from 8 to $1024 \mu \mathrm{g} / \mathrm{ml}$. Each bacterial suspension (1 $\mu \mathrm{l})$ was inoculated $\left(3 \times 10^{5} \mathrm{CFU} / \mathrm{spot}\right)$ on drug containing plates. Plates with no drugs were inoculated at the beginning and end of each run as controls.

To determine the possible effect of aspirin on the MICs of amoxycillin, metronidazole, and clarithromycin, susceptibility testing of these three widely used antimicrobials in the presence or absence of aspirin were performed using the E test and agar dilution methods. Aspirin at a final concentration of 1 $\mathrm{mM}(180 \mu \mathrm{g} / \mathrm{ml})$ was added to Columbia blood agar to obtain aspirin containing plates. Each bacterial suspension $(100 \mu \mathrm{l})$ was spread on plates with or without aspirin using a cotton swab. A single E test drug strip (AB Biodisk, Sweden) was applied to the surface of each dried plate. For each strain, MICs obtained in the presence or absence of aspirin were compared. E coli (DH5a) was included in this assay.

\section{Statistical analysis}

Time killing studies of $H$ pylori were performed using five different strains. Representative data from these experiments are presented as mean (SEM). The Student's $t$ test was used to compare data. A p value of less than 0.05 was considered statistically significant.

\section{RESULTS}

Effects of aspirin and salicylate on the growth of

\section{$H$ pylori}

Strains of $H$ pylori were inoculated in broth medium containing various concentrations of aspirin $(0,200,300$, and 400 $\mu \mathrm{g} / \mathrm{ml})$ and salicylate $(0,0.56,1.1,1.6$, and $2.2 \mathrm{mM})$. The effects on the growth of bacteria were determined spectrophotometrically as well as by a viable count method at 0, 24, and 48 hours. Similar results were obtained by performing experiments with five different strains, indicating that aspirin inhibited the growth of $H$ pylori in a dose dependent manner. As determined by optical density at $600 \mathrm{~nm}$, aspirin at a concentration of $100 \mu \mathrm{g} / \mathrm{ml}$ began to show an inhibitory effect on the growth of $H$ pylori compared with the vehicle control. Concentration of aspirin of $400 \mu \mathrm{g} / \mathrm{ml}$ stopped increases in optical density, indicating cessation of cell growth. Further tests, based on determining the numbers of bacteria that could form colonies, showed that aspirin at a concentration of $400 \mu \mathrm{g} / \mathrm{ml}$ was lethal, resulting in a 1 log reduction in the numbers of $\mathrm{CFU} / \mathrm{ml}$ relative to the numbers in the inoculum at 24 hours, and produced nearly a $2 \log$ decrease at 48 hours (fig lA). The experiment was repeated using equivalent concentration of salicylate and the results were essentially similar to those of aspirin (fig 1B). Morphological studies performed by Gram staining revealed complete lysis of cells incubated with 400 $\mu \mathrm{g} / \mathrm{ml}$ of aspirin for 48 hours whereas no such lysis was detected in control cultures (fig 2). No significant coccoid formation was found after aspirin treatment. 

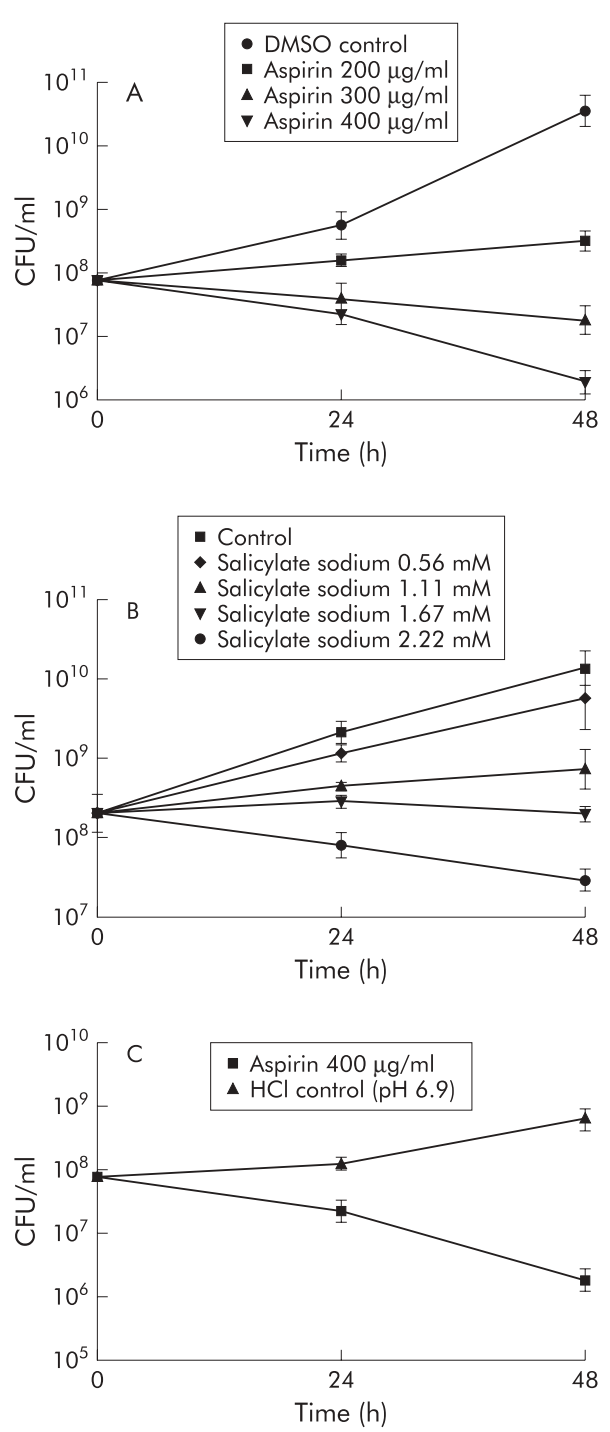

Figure 1 Inhibitory effect of aspirin and salicylate on the growth of Helicobacter pylori. (A) Aspirin treatment; (B) salicylate treatment. Data are representative of the average of five independent experiments using different strains. (C) Growth of $H$ pylori strain NCTC 11637 in the presence $(\mathrm{pH}$ dropped from 7.2 to 6.9 after aspirin) and absence (pH titrated to 6.9 by $1 \mathrm{~N} \mathrm{HCl}$ ) of aspirin. All results are expressed as the mean (SEM) of at least three independent experiments. DMSO, dimethylsulphoxide; CFU, colony forming unit.

\section{Effects of decreased $\mathrm{pH}$ on the growth of $\boldsymbol{H}$ pylori}

The $\mathrm{pH}$ of Brucella broth supplemented with 10\% FBS before inoculation was 7.26 whereas the $\mathrm{pH}$ of the medium after adding $400 \mu \mathrm{g} / \mathrm{ml}$ of aspirin was 6.90 . However, growth of $H$ pylori NCTC 11637 in the presence of $400 \mu \mathrm{g} / \mathrm{ml}$ aspirin was 2 logs lower than that in the absence of the drug at the same $\mathrm{pH}$ after 48 hours of incubation (fig $\mathrm{lC}$ ). Thus aspirin does not exert its effect by changing the $\mathrm{pH}$ of the medium.

\section{Synergism between metronidazole and aspirin}

Adding aspirin to Columbia blood agar medium caused marked decreases in colony size compared with controls. No rdxA or frxA dependent differences in efficiency of plating or colony size as a function of aspirin dose were detected. The efficiency of plating decreased more dramatically with doses of 16,25 , or $32 \mu \mathrm{g} / \mathrm{ml}$ of metronidazole with aspirin $100 \mu \mathrm{g} / \mathrm{ml}$, and especially with aspirin $200 \mu \mathrm{g} / \mathrm{ml}$, compared with aspirin free plates. Thus aspirin and metronidazole were strongly synergistic with respect to inhibition of $H$ pylori colony formation.
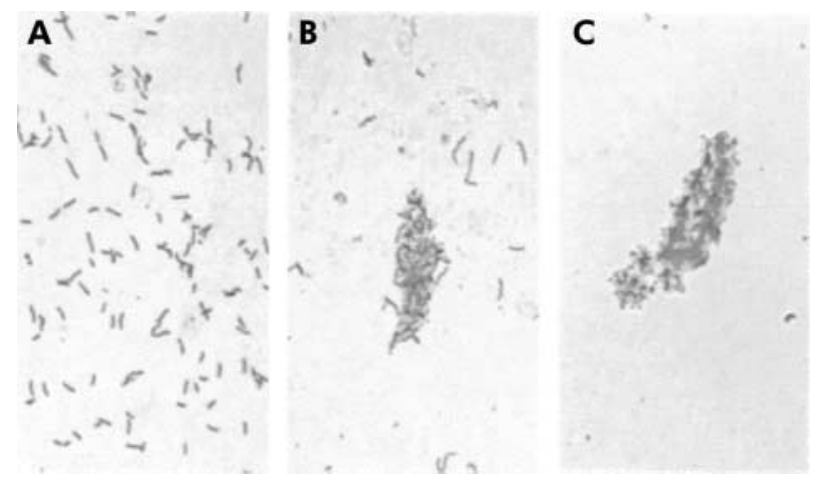

Figure 2 Gram staining of Helicobacter pylori strain NCTC 11637 . (A) Vehicle control at 48 hours. (B) Treated with $200 \mu \mathrm{g} / \mathrm{ml}$ aspirin at 48 hours. (C) Treated with $400 \mu \mathrm{g} / \mathrm{ml}$ aspirin at 48 hours. Magnifications $\times 1000$.

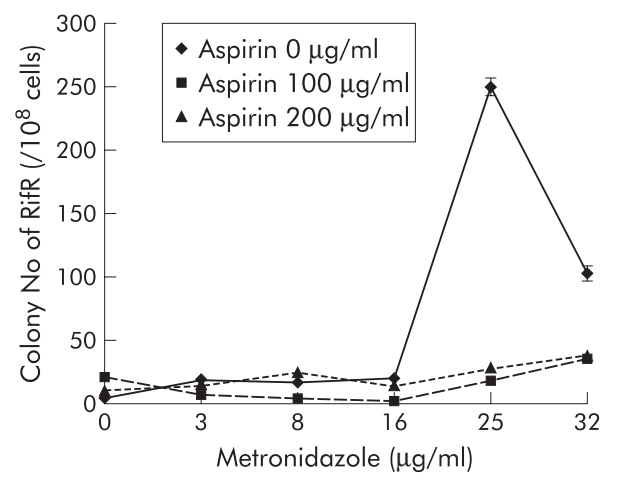

Figure 3 Inhibitory effect of aspirin on metronidazole induced mutation to rifampicin resistance (RifR) after three days of incubation in Columbia agar containing various concentrations of metronidazole and aspirin. All results are expressed as the mean (SEM) of three independent experiments .

\section{Effect of aspirin on metronidazole induced mutation}

Inclusion of inhibitory levels of aspirin ( 100 or $200 \mu \mathrm{g} / \mathrm{ml}$ ) in blood agar medium suppressed the normal ability of metronidazole at 25 or $32 \mu \mathrm{g} / \mathrm{ml}$ to induce new mutations to RifR (fig 3).

\section{MICs of aspirin}

The MICs of aspirin for $66 \mathrm{H}$ pylori strains were tested. The $\mathrm{MIC}_{50}$ of aspirin was $256 \mu \mathrm{g} / \mathrm{ml}, \mathrm{MIC}_{90}$ was $512 \mu \mathrm{g} / \mathrm{ml}$, and the range of MIC values was $256-512 \mu \mathrm{g} / \mathrm{ml}$. There were no differences regarding MICs against reference strains and clinical isolates derived from patients taking or not taking aspirin.

MICs of amoxycillin, metronidazole, and clarithromycin in the presence of aspirin

Susceptibility testing of three widely used antimicrobial agents (amoxycillin, metronidazole, and clarithromycin) in $H$ pylori eradication was performed on 24 strains in the presence of aspirin at a final concentration of $1 \mathrm{mM}(180 \mu \mathrm{g} / \mathrm{ml})$ and compared with that in the absence of aspirin. Aspirin decreased MICs in all strains $(24 / 24,100 \%)$ tested for amoxycillin, and 18 strains $(18 / 24,75 \%)$ tested for metronidazole and clarithromycin, indicating increased susceptibility of $H$ pylori to these drugs. More $\log _{2}$ decreases in MICs were found for metronidazole compared with clarithromycin (3.75 (0.77) $v 1.87$ (0.46); $\mathrm{p}=0.04$ ) (fig 4). It was difficult to determine the exact value of the $\log _{2}$ decrease in MIC for amoxycillin as $H$ pylori, in the presence or absence of aspirin, was very susceptible to amoxycillin. For the six resistant strains (four resistant to metronidazole only, one resistant to both metronidazole and clarithromycin, and one resistant to clarithromycin only), 


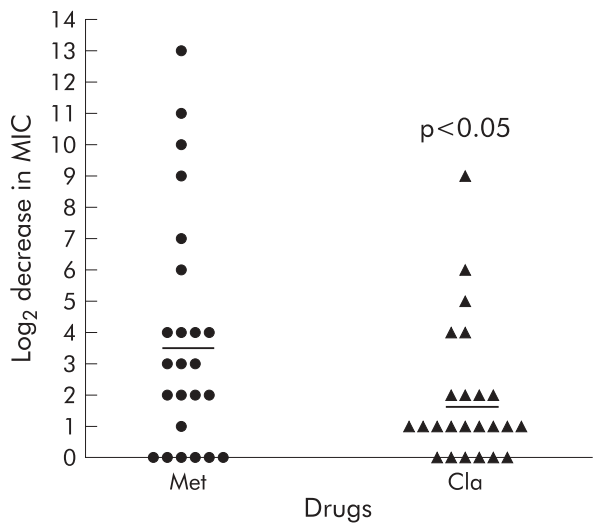

Figure $4 \log _{2}$ decrease in the minimal inhibitory concentration (MIC) of metronidazole (Met) and clarithromycin (Cla) in the presence of aspirin in 24 Helicobacter pylori strains. Greater $\log _{2}$ decreases in MICs were found for metronidazole compared with clarithromycin (3.75 (0.77) v. $1.87(0.46) ; p=0.04)$. Horizontal bars indicate mean $\log _{2}$ decreases.

Table 1 Minimal inhibitory concentrations for the six representative strains in the presence or absence of aspirin

\begin{tabular}{llllll}
\hline \multirow{2}{*}{$\begin{array}{l}\text { No of } \\
\text { strains }\end{array}$} & \multicolumn{2}{l}{ Metronidazole } & & \multicolumn{2}{l}{ Clarithromycin } \\
\cline { 2 - 3 } \cline { 5 - 6 } \cline { 5 - 6 } & No aspirin & Aspirin & & No aspirin & Aspirin \\
\hline 1 & 128 & 0.25 & & 0.016 & 0.016 \\
2 & 256 & 0.25 & & 128 & 2 \\
3 & 64 & 0.5 & & 0.032 & 0.016 \\
4 & 256 & 0.032 & & 0.064 & 0.016 \\
5 & 32 & 0.016 & & 0.25 & 0.125 \\
6 & 4 & 0.25 & & 16 & 0.032 \\
\hline
\end{tabular}

MICs decreased significantly in the presence of aspirin (table 1). Aspirin converted five strains from resistant to susceptible. In contrast, the MICs of these drugs increased in the presence of aspirin when $E$ coli was used as the testing strain under the same conditions.

\section{DISCUSSION}

Although there is great interest in the role of $H$ pylori infection and aspirin usage in peptic ulcer diseases, information on the mechanism of interaction between the two factors is lacking. $H$ pylori may provide some protection against aspirin related ulcers due to its ability to stimulate mucosal synthesis of prostaglandins, ${ }^{2-4}$ or aspirin may influence the pathogenic effects of $H$ pylori as a result of possible effects on this microorganism. In the present study, we found that aspirin and salicylate significantly inhibited the growth of $H$ pylori in vitro, and reduced the efficiency of colony formation and colony size. Growth inhibition was found in all strains tested, including all clinical isolates, two reference strains (NCTC 11637, NCTC 12908), H pylori 26695 and SS1, and their isogenic MtzR derivatives (rdxA::cam and frxA::kan single and double knockout mutants). This indicates that the inhibitory effect of aspirin is universal among $H$ pylori. No rdxA or frxA dependent differences in efficiency of colony formation or colony size in relation to aspirin dose were found. The fact that aspirin inhibited the growth of $\mathrm{H}$ pylori was in accordance with previous studies, which showed that aspirin could inhibit the growth of other bacterial species. ${ }^{8-12}$

Some have postulated that the coccoid form is a dormant stage of $H$ pylori used for survival in hostile environment ${ }^{19-21}$ whereas others have argued that coccoid forms are simply dead or dying. ${ }^{22}$ In any case, no coccoid formation was induced by the stress of aspirin treatment, not associated with the lethality of higher doses. There was a tendency for $\mathrm{H}$ pylori to form clusters when treated with aspirin. The reason for this is uncertain but may be related to changes in membrane properties (see below).

$H$ pylori can survive in vitro in a wide $\mathrm{pH}$ range of 4.0 8.0. ${ }^{23-27}$ Aspirin $\left(\mathrm{p} K_{\mathrm{a}} 3.5\right)$ is a weak acid, ${ }^{28}$ leading to a $\mathrm{pH}$ drop from 7.26 to 6.90 when added to the medium at $400 \mu \mathrm{g} / \mathrm{ml}$. Growth of $H$ pylori was inhibited significantly in the presence of aspirin compared with controls at the same $\mathrm{pH}$. This showed that the inhibitory effect of aspirin on the growth of $H$ pylori was not solely due to its ability to act as a weak acid.

A plasma level of $20-100 \mu \mathrm{g} / \mathrm{ml}$ of aspirin is recommended for analgesia and $150-300 \mu \mathrm{g} / \mathrm{ml}$ for an anti-inflammatory effect. ${ }^{28}$ Relatively high concentrations of aspirin are used for pain and fever, and in rheumatic fever, gout, and rheumatoid arthritis. ${ }^{29}$ In our study, the inhibitory effect of aspirin on $H$ pylori was observed at $100 \mu \mathrm{g} / \mathrm{ml}$ and the increasing susceptibility to amoxycillin, clarithromycin, and metronidazole was induced by aspirin at $1 \mathrm{mM}(180 \mu \mathrm{g} / \mathrm{ml})$. Therefore, the effective concentrations of aspirin on $H$ pylori in vitro are comparable with normal plasma levels. These concentration of aspirin may be reached only transiently because it is rapidly metabolised to salicylate. We repeated the experiment using salicylate at a concentration of $0.56,1.1,1.6$, and $2.2 \mathrm{mM}$ (equivalent to aspirin $100,200,300$, and $400 \mu \mathrm{g} / \mathrm{ml}$, respectively) and the results were essentially similar (fig 1B). A plasma salicylate level of 1.4-1.8 mM is considered therapeutic and plasma levels greater than $2.2 \mathrm{mM}$ are potentially toxic in chronic salicylate dosing. Thus the effect of aspirin and salicylate demonstrated in our study is achievable in vivo.

Although the mechanism by which aspirin inhibits the growth of $H$ pylori and increases susceptibility to amoxycillin, clarithromycin, and metronidazole was not investigated in this study, the efficiency of plating decreased with high doses of metronidazole in the presence of aspirin. This apparent synergism between aspirin and metronidazole reflects in part the reduction in colony size to the point of invisibility but might be distinct from outright killing caused by bactericidal concentrations of metronidazole itself. ${ }^{16}$

Metronidazole at partially inhibitory concentrations causes mutation in growing $\mathrm{H}$ pylori due to conversion of this prodrug to hydroxylamine and related compounds that are both bactericidal and mutagenic. It is remarkable that aspirin treatment suppressed this normal mutagenic effect of metronidazole on $H$ pylori even though it exacerbated its lethality. This implies that these two drugs act in different manners, and suggests models in which incipient mutant bacteria which have been weakened by metronidazole exposure may be killed preferentially by aspirin.

We then quantified new mutations to RifR in the presence or absence of aspirin, as a measure of metronidazole induced mutation in $H$ pylori. The results showed clearly that inclusion of inhibitory levels of aspirin suppressed, rather than enhanced, the normal ability of metronidazole at $25 \mu \mathrm{g} / \mathrm{ml}$ or $32 \mu \mathrm{g} / \mathrm{ml}$ to induce new mutations to RifR. These data suggest that the inhibitory effect of aspirin on $\mathrm{H}$ pylori involves a mechanism distinct from DNA base modification and probably strand breakage also, the types of damage that underlie the mutagenic and bactericidal effects of metronidazole on this gastric pathogen. ${ }^{30}$

There has been extensive study of the interaction between aspirin and various antibiotics in organisms such as $E$ coli. Aspirin or salicylate increase the resistance of $E$ coli to several negatively charged or neutral antibiotics, including ampicillin, tetracycline, chloramphenicol, nalidixic acid, and cephalosporins..$^{31}{ }^{32}$ One mechanism of this resistance entails aspirin induced downregulation of expression of the outer membrane porin $\mathrm{OmpF}^{33-35}$ which serves as a channel for the entry of these antibiotics into the periplasmic space. ${ }^{36}$ 
Salicylate decreased the amount of OmpF by two means, one dependent on and one independent of the much studied multiple antibiotic resistant gene in $E$ coli. ${ }^{34}{ }^{35}{ }^{37}$ In clinical isolates this may result from stepwise mutations that may revert when antibiotic selection pressure is removed. In addition, aspirin is a weak acid and could increase the membrane potential of species such as E coli, Salmonella typhimurium, and Pseudomonas aeruginosa, and thereby enhance the susceptibility to drugs such as novobiocin and aminoglycoside antibiotics. ${ }^{13}{ }^{14}$ Salicylates can also increase the uptake of divalent ions such as cadmium in $E$ coli, potentiating $\mathrm{Cd}^{2+}$ toxicity, independent of an increase in membrane potential..$^{38}$ In this study, we observed increased susceptibility to amoxycillin, clarithromycin, and metronidazole of $H$ pylori in the presence of aspirin. Whether the above mechanisms are true for $H$ pylori remains to be determined.

Three results indicate that the increased susceptibility to amoxycillin, clarithromycin, and metronidazole caused by aspirin was not solely due to changes in $\mathrm{pH}$ in aspirin containing medium. Firstly, the $\mathrm{pH}$ of the Columbia agar medium decreased from 7.20 to 7.08 with $1 \mathrm{mM}$ aspirin, and thus this $\mathrm{pH}$ change did not interfere significantly with the growth of $H$ pylori. ${ }^{40}$ Secondly, the bactericidal activity of amoxycillin and clarithromycin require high growth rate and high expression of bacterial targets at neutral $\mathrm{pH}$, and increased resistance to these antibiotics will be observed for the poor growth or non-dividing state of bacteria at acidic $\mathrm{pH}^{24}{ }^{24} 42$ Thirdly, metronidazole, a DNA targeted antibiotic, does not depend on bacterial cell division for its activity. Therefore, the activity of metronidazole is not $\mathrm{pH}$ dependent and remains stable. ${ }^{24}{ }^{413}$ This was in accordance with our finding that the $\log _{2}$ decrease in MIC of metronidazole was more frequently observed than that of amoxycillin and clarithromycin in the presence of aspirin.

In conclusion, our results provide evidence that aspirin inhibits the growth of $H$ pylori and increases the susceptibility to antimicrobial agents in vitro. How well this in vitro activity translates to in vivo effectiveness either in clearing the infection or in suppressing metronidazole induced mutation (that may be important in virulence or drug resistance) merits further analysis in clinical and experimental animal infection studies. This mechanism is therefore important in future drug development for effective clearing and overcoming resistance.

\section{ACKNOWLEDGEMENT}

This study was supported in part by the Peptic Ulcer Research Fund, the Simon KY Lee Gastroenterology Research Fund, University of Hong Kong, and US Public Health Service grants AI38166, AI49161, DK53727, and P30 DK52574. The authors thank senior technician Ms E Kwok and endoscopy nurses Ms M. Chong, KW Wong, VSY Tang, MY Lee, and KK Chang for assistance, and Dr Sidney Tam of Clinical Biochemistry Unit, Queen Mary Hospital, for his advice on the manuscript.

\section{Authors' affiliations}

W H Wang*, Q Gu, Department of Gastroenterology, First Hospital, Peking University, Beijing, China

W M Wong*, K C Lai, S K Lam, B C Y Wong, Department of Medicine, University of Hong Kong, Hong Kong, China

D Dailidiene, D E Berg, Department of Molecular Microbiology,

Washington University Medical School, St Louis, Missouri 63110, USA

*W H Wang and W M Wong contributed equally to this work

\section{REFERENCES}

1 Kordecki H, Kurowski M, Kosik R, et al. Is Helicobacter pylori infection a risk or protective factor for mucosal lesions development in patients chronically treated with acetylsalicylic acid? J Physiol Pharmacol 1997:48(suppl 4):85-91.

2 Cryer B, Feldman M. Effects of very low dose daily, long-term aspirin therapy on gastric, duodenal, and rectal prostaglandin levels and on mucosal injury in healthy humans. Gastroenterology 1999;117:7-25.
3 Hudson N, Balsitis M, Filipowicz F, et al. Effect of Helicobacter pylori colonisation on gastric mucosal eicosanoid synthesis in patients taking nonsteroidal anti-inflammatory drugs. Gut 1993;34:748-51.

4 Laine L, Cominelli F, Sliane R, et al. Interaction of NSAIDs and Helicobacter pylori on gastrointestinal injury and prostaglandin production: a controlled double-blind trial. Aliment Pharmacol Ther 1995; 9: 127-35.

5 Caselli M, Pazzi P, LaCorte R, et al. Campylobacter-like organisms, nonsteroidal anti-inflammatory drugs and gastric lessions in patients with rheumatoid arthritis. Digestion 1989:44:101-4.

6 Graham DY, Lidsky MD, Cox AM, et al. Long-term nonsteroidal anti-inflammatory drug use and Helicobacter pylori infection. Gastroenterology 1991;100:1653-7.

7 Loeb DS, Talley NJ, Ahlquist DA, et al. Long-term nonsteroidal anti-inflammatory drug use and gastroduodenal injury: the role of Helicobacter pylori. Gastroenterology 1992;102:1899-905.

8 Cederlund H, Mardh PA. Antibacterial activities of non-antibiotic drugs. J Antimicrob Chemother 1993;32:355-65.

9 Cederlund H, Mardh P-A. Antimicrobial activities of N-acetylcysteine and some non-steroidal antiinflammatory drugs. J Antimicrob Chemother 1993;32:903-4.

10 Burns JL, Hedin LA, Lien DM. Chloramphenicol resistance in pseudomonas cepacia because of decreased permeability. Antimicrob Agent Chemother 1989;33:136-41.

11 Burns JL, Clark DK. Salicylate-inducible antibiotic resistance in Pseudomonas cepacia associated with absence of a pore-forming outer membrane protein. Antimicrob Agent Chemother 1992;36:2280-5.

12 Price CTD, O'Brien FG, Shelton BP, et al. Effects of salicylate and related compounds on fusidic acid MICs in Staphylococcus aureus. J Antimicrob Chemother 1999;44:57-64.

13 Ohsuka S, Ohta M, Masuda K, et al. Lidocaine hydrochloride and acetylsalicylate kill bacteria by disrupting the bacterial membrane potential in different ways. Microbiol Immunol 1994;38:429-34.

14 Aumercier M, Murray DM, Rosner JL. Potentiation of susceptibility to aminoglycosides by salicylate in Escherichia coli. Antimicrob Agent Chemother 1990;34:786-91.

15 Graham DY, Klein PD, Opekun AR, et al. In vivo susceptibility of Campylobacter pylori. Am J Gastroenterol 1989;84:233-8.

16 Jeong JY, Mukhopadhyay AK, Akada JK, et al. Roles of FrxA and RdxA nitroreductases of Helicobacter pylori in susceptibility and resistance to metronidazole. J Bacteriol 2001; 183:5155-62.

17 Heep $M$, Beck D, Bayerdorffer E, et al. Rifampin and rifabutin resistance mechanism in Helicobacter pylori. Antimicrob Agent Chemother 1999:43: 1497-9.

18 National Committee for Clinical Laboratory Standards (NCCLS). Performance Standards for Antimicrobial Susceptibility testing, Fourth Information Supplement. NCCLS Document M100-S4, Villanova, PA, 1992

19 Benaissa M, Babin P, Quellard N, et al. Changes in Helicobacter pylori ultrastructure and antigens during conversion from the bacillary to the coccoid form. Infect Immun 1996;64:2331-5

20 Bode G, Mauch F, Malfertheiner P. The coccoid forms of Helicobacter pylori. Criteria for their viability. Epidemiol Infect 1993;111:483-90.

21 Shahamat M, Mai U, Paszko-Kova C, et al. Use of autoradiography to assess viability of Helicobacter pylori in water. Appl Environ Microbiol 1993:59:1231-5.

22 Eaton KA, Catrenich CE, Makin KM, et al. Virulence of coccoid and bacillary forms of Helicobacter pylori in gnotobiotic piglets. J Infect Dis 1995; 171:459-62.

23 Clyne M, Labigne A, Drumm B. Helicobacter pylori requires an acidic environment to survive in the presence of urea. Infect Immun 1995;63:1669-73

24 Scott D, Weeks D, Melchers K, et al. The life and death of Helicobacter pylori. Gut 1998;43:S56-60.

25 Goodwin CS, Collin MD, Blincow E. The absence of thermoplasmaquinones in Campylobacter pyloridis, and its temperature and $\mathrm{pH}$ growth range. Microbios Lett 1986;32:137-40.

26 Goodwin CS, Armstrong JA. Microbiological aspects of Helicobacter pylori (Campylobacter pylori). Eur J Clin Microbiol Infect Dis 1990;9:1-13.

27 Meyer-Rosberg K, Scott DR, Rex D, et al. The effect of environmental pH on the proton motive force of Helicobacter pylori. Gastroenterology 1996; 111:886-900

28 Moffat AC, Jackson JV, Moss MS, et al. Clarke's Isolation and Identification of Drugs in Pharmaceuticals, Body Fluids, and Post-mortem Material. London: Pharmaceutical Press, 1986:361-2.

29 Meissmann G. Aspirin. Sci Am 1991.264.58-64

30 Sisson G, Jeong JY, Goodwin A, et al. Metronidazole activation is mutagenic and causes DNA fragmentation in Helicobacter pylori and in Escherichia coli containing a cloned $H$. pylori $\mathrm{RdxA}(+)$ (nitroreductase) gene. J Bacteriol 2000;182:5091-6.

31 Foulds J, Murray DM, Chai T, et al. Decreased permeation of cephalosporins through the outer membrane of Escherichia coli grown in salicylates. Antimicrob Agent Chermother 1989;33:412-17.

32 Rosner JL. Nonheritable resistance to chloramphenicol and other antibiotics induced by salicylates and other chemotactic repellents in Escherichia coli K-12. Proc Natl Acad Sci U S A 1985;82:8771-4.

33 Rosner JL, Chai T, Foulds J. Regulation of OmpF porin expression by salicylate in Escherichia coli. J Bacterial 1991;173:5631-8.

34 Cohen SP, McMurry LM, Levy SB. mar locus causes decreased expression of OmpF porin in multiple-antibiotic-resistant (Mar) mutants of Escherichia coli. J Bacteriol 1988;170:5416-22. 
35 Cohen SP, Levy SB, Foulds J, et al. Salicylate induction of antibiotic resistance in Escherichia coli: activation of the mar operon and a mar-independent pathway. J Bacteriol 1993;175:7856-62.

36 Nikaido H, Vaara M. Molecular basis of bacterial outer membrane permeability. Microbiol Rev 1985;49:1-32.

37 Cohen SP, Hachler H, Levy SB. Genetic and functional analysis of the multiple antibiotic resistance (mar) locus in Escherichia coli. J Bacteriol 1993:175: 1484-92.

38 Rosner JL, Aumercier M. Potentiation by salicylate and salicyl alcohol of cadmium toxicity and accumulation in Escherichia coli. Antimicrob Agent Chemother 1990;34:2402-6.

39 Merlin TL, Davis GE, Anderson WL, et al. Aminoglycoside uptake increased by tet gene expression. Antimicrob Agent Chemother 1989;33:230-2.
40 Morgan DR, Freedman R, Depew CE, et al. Growth of Campylobacter pylori in liquid media. J Clin Microbiol 1987;25:2123-5.

41 Sjöström JE, Laesson $\mathrm{H}$. Factors affecting growth and antibiotic susceptibility of Helicobacter pylori: effect of $\mathrm{pH}$ and urea on the survival of a wild-type strain and a urease- deficient mutant. J Med Microbiol 1996:44:425-33.

42 Malanoski GJ, Eliopoulos GM, Ferraro M, et al. Effect of $\mathrm{pH}$ variation on the susceptibility of Helicobacter pylori to three macrolide antimicrobial agents and temafloxacin. Eur J Clin Microbiol Infect Dis 1993;12:131-3.

43 Grayson ML, Eliopoulos GM, Ferraro M, et al. Effect of varying pH on the susceptibility of Campylobacter pylori to antimicrobial agents. Eur $J$ Clin Microbiol Infect Dis 1989;8:888-9.

\section{Gut through the ages}

\section{Browse the Archive}

Gut online has an archive of content dating back to 1966 .

Full text from 1997; abstracts from 1975; table of contents from 1966

www.gutjnl.com 